\title{
Fachdidaktiken im Spannungsfeld zwischen kompetenzorientiertem fachlichen Lernen und inklusiver Pädagogik: Vereinigungsbemühungen oder Verdeckungsgeschehen?
}

\author{
Michaela Vogt $\cdot$ Till Neuhaus
}

Eingegangen: 4. Dezember 2020 / Angenommen: 18. Dezember 2020 / Online publiziert: 12. Januar 2021 (C) Der/die Autor(en) 2021

Zusammenfassung In den vergangenen 20 Jahren sahen sich die Fachdidaktiken zwei zentralen paradigmatischen Wendungen ausgesetzt: der durch PISA (2000) induzierten Kompetenzorientierung und der Umsetzung der Behindertenrechtskonvention der Vereinten Nationen (2006/2009). Auf ihre historischen Wurzeln zurückgeführt verhalten sich diese beiden Ansätze antinomisch zueinander und erzeugen für die Fachdidaktiken ein Spannungsfeld, in dem sich diese positionieren und verorten müssen. Dieser Beitrag argumentiert, dass die bis dato dominante Kompetenzorientierung auf dieses neu entstandene Spannungsfeld mit einem Verdeckungsgeschehen antwortete - einer Denkfigur, die bereits in einigen geistes- und sozialwissenschaftlichen (Teil-)Disziplinen beobachtet, aber noch nie metatheoretisch systematisiert worden ist. Anhand der Bereiche Teilhabe, Leistung und Perspektive (auf Schüler) konkretisiert der Beitrag diese Überlegungen.

Schlüsselwörter Kompetenz · Fachdidaktik · Inklusive Pädagogik · Verdeckung

Prof. Dr. M. Vogt $(\varangle)$

Professur für Erziehungswissenschaft mit dem Schwerpunkt Theorie und Geschichte der Inklusiven Pädagogik, Fakultät für Erziehungswissenschaften, Universität Bielefeld, Universitätsstraße 25, 33501 Bielefeld, Deutschland

E-Mail: michaela.vogt@uni-bielefeld.de

T. Neuhaus

Fakultät für Erziehungswissenschaften, Universität Bielefeld, Universitätsstraße 25, 33501 Bielefeld, Deutschland

E-Mail: till.neuhaus@uni-bielefeld.de 


\title{
Subject-based didactics oscillating between the demands of competence- based learning and inclusive pedagogy: unification or concealment of incompability?
}

\begin{abstract}
In the past 20 years, subject-oriented didactics saw themselves confronted with two major paradigms: The, induced by PISA (2000), turn towards competencebased learning and the realization of the United Nation's Convention on the Rights of Persons with Disabilities (2006/2009). Due to their specific historical developments, these two approaches can be considered antinomical and their co-existence causes tensions for the subject-oriented didactics. This article argues that the-up to this point dominant - approach of competence-based learning react towards this disruption with a "Verdeckungsgeschehen" (cloak of silence), a reaction which has been observed in a variety of social sciences, yet it has never been conceptualized on a metalevel. By focusing on the areas of participation, performance, and perspective (on students), this article reflects on this idea.
\end{abstract}

Keywords Competences $\cdot$ Didactics $\cdot$ Inclusive pedagogy $\cdot$ Cloak of silence

\section{Einleitung}

Eine Funktion von Schule ist die Vermittlung von gesellschaftlich relevanten Werten und Normen an die nachwachsende Generation. Die in der Schule realisierten Werte manifestieren sich in verschiedenster Form, sei es in der Architektur des Gebäudes, die Ausgestaltung von Schüler-Lehrer-Beziehungen, curriculare Inhalte u. v. m. (vgl. Hummrich und Kramer 2017, S. 8 ff.; vgl. Jackson 1968). Eine besondere Rolle in diesem Kontext nehmen dabei die Fachdidaktiken ${ }^{1}$ ein, da sie als zentraler Baustein das fachliche Lernen strukturieren und - sowohl durch Modellbildung wie auch durch konkrete Handlungsempfehlungen - große Teile der schulischen und unterrichtlichen Prozesse beeinflussen. Daher sind besonders die Fachdidaktiken an der Realisierung von gesellschaftlichen Werten beteiligt, die sich u.a. in Kernlehrplänen, Ausbildungsverordnungen und Studieninhalten widerspiegeln. Insofern waren sie ebenfalls in die Implementation der UN-BRK (2009) in den schulischen Kontext involviert, als sich damit ,die Frage inklusiver Pädagogik und Didaktik [...] von einem vormals enggeführten Spezialdiskurs zu einem breiteren Interdiskurs über schulische Bildung“ (Schroeder 2017, S. 255) wandelte.

Die Umsetzung bzw. Verhandlung neuer Diskurslinien wie die der Inklusion ist dabei für die Grundschule von besonderer Bedeutung, da sie ,im deutschen Schulsystem die Schulform [ist], in der die Vorstellung einer Schule für alle Kinder ihren konsequenten Ausdruck findet" (Götz et al. 2016, S. 3). Nach über einem Jahrzehnt intensivster Bemühungen, inklusive Pädagogik - in der Grundschule sowie in den weiterführenden Schulen - im Generellen sowie ebenso im Spezifischen in

\footnotetext{
1 Fachdidaktiken werden als jene praktische und wissenschaftliche Disziplin konzeptualisiert, die fachliches Lernen in der Institution Schule erforscht. Jenseits dieser allgemeinen Definition kann der vorliegende Artikel nicht auf die Spezifika einzelner Fachdidaktiken bspw. auf konzeptioneller Ebene eingehen.
} 
den Fachdidaktiken zu installieren, fällt die Bilanz gerade hinsichtlich des letztgenannten Aspekts eher ernüchternd aus. So attestiert Ritter (2021, in dieser Ausgabe) hinsichtlich deren Verhältnisses zur inklusiven Pädagogik Folgendes:

Es scheint sich also in den Fachdidaktiken eine Diskussion zu entwickeln, die das Thema oberflächlich adaptiert, dahinter aber ein eigenes Begriffskonstrukt hervorbringt und die eigenen Konzepte inklusiver Didaktik kaum systematisch mit dem paradigmatischen Verständnis der inklusiven Pädagogik verzahnt. Das zeigt sich im Übrigen auch auf den entsprechenden Treffen der Disziplinen. Auf einer fachdidaktischen Tagung treten Inklusionpädagog*innen bestenfalls als einführende Hauptredner*innen auf, auf einer Tagung der Inklusionspädagogik finden sich nur selten Fachdidaktiker*innen.

Anknüpfend an die Beobachtungen Ritters (2021) fragt der vorliegende Text nach den Gründen für diesen Status quo und möchte hier v. a. auf das antinomische Verhältnis von inklusiven Ansprüchen und kompetenzorientiertem fachlichen Lernen als zentrale Herausforderung auch für die Fachdidaktiken in den letzten 20 Jahren eingehen. Zeitlich kann das breite Aufkommen dieser beiden Bezugsgrößen bzw. ihre Relevanzsetzung seitens der Politik weitgehend klar bestimmt werden. Für die Kompetenzorientierung im bundesdeutschen Raum spielen hier Reformen, die als Antwort auf den vermeintlichen PISA-Schock (2000) umgesetzt worden sind, eine zentrale Rolle. Allerdings muss dabei bedacht werden, dass Kompetenzorientierung als dominierender Ansatz bereits seit den 1960er-Jahren global existiert, jedoch erst Anfang der 2000er Jahre über internationale Vergleichsstudien und Rankings seinen Einzug auch in deutsche Lehrpläne gefunden und damit hier zu einem Wandel im Bereich Schule geführt hat (vgl. Tröhler 2019). Auch Vorläuferentwicklungen inklusiver Pädagogik stellen bildungshistorisch betrachtet eine Form der Vorgeschichte zu den seit der UN-BRK national verstärkt nachweisbaren Inklusionsbemühungen dar (vgl. Vogt et al. im Druck). Auf Grundlage dieser beiden parallel bestehenden und bereits in ihrer internen Logik divergierenden Ansprüche entsteht ein Spannungsfeld, dessen jeweiligen Pole um die Ausrichtung der Fachdidaktiken ringen.

In Orientierung an diese Ausgangslage hat es sich der vorliegende Beitrag zum Ziel gesetzt, alternative Erklärungen für die von Ritter (2021) gemachten Beobachtungen zu präsentieren und zur Diskussion zu stellen. Dafür nimmt der Beitrag v. a. eine bildungshistorische Perspektive ein, die ideen- wie sozialgeschichtliche Elemente aufgreift und zeichnet so die Genese der aufgeführten Argumentationsfiguren in den letzten 20 Jahren - lediglich exemplarisch und in ihrer basalsten Form nach. Anschließend wird die Denkfigur des Verdeckungsgeschehens dargestellt und über die Bereiche Teilhabe, Leistung und Perspektive reflexiv an die Fachdidaktiken zurückgebunden. Fachdidaktiken stellen sozusagen das Medium dar, in dem sich der oben skizzierte und in Abschn. 2 ausformulierte Paradigmenstreit manifestiert. 


\section{Inklusion und Kompetenzorientierung als antinomische Ansprüche an die Fachdidaktiken}

\subsection{Bildungsstandards, PISA und die nationale Sicherheit}

Die Geschichte des kompetenzorientierten fachlichen Lernens lässt sich bis in die 1960er-Jahre zurückverfolgen. Am 4. Oktober 1957 gelang es der Sowjetunion den Satelliten Sputnik 1 ins All zu befördern - ein Ereignis, das im Westen und im Besonderen in den USA den sogenannten Sputnikschock auslöste. Im Wettbewerb der (Wirtschafts-)Systeme verbesserten die sozialistischen Staaten damit ihre Position beim Wettlauf ins All. Die Reaktion der USA wurde sinnbildlich vom damaligen Vizepräsidenten Richard Nixon verbalisiert: ,If the free world is to survive we cannot rest on our present position of military superiority. We must constantly push forward on all fronts" (zit. in Creagh 1957). Eine dieser Fronten, die vom verantwortlichen Planer - dem Navy Admiral und Verantwortlichen für die Nuklearmarine H.G. Rickover - als first line of defense (1959a) konzeptualisiert wurde, war der Bildungsbereich. Die dort stattfindende Umstrukturierung hatte vornehmlich das Ziel zukünftig die Ausbildung von Naturwissenschaftlern und Forschern zu fokussieren, wie das folgende Zitat (Coombs 1958 zit. in Buss 1980, S. 339) illustriert:

By honoring excellence of performance in education and by honoring quality in education the Federal Government can do much to help expand the Nation's total supply of well-developed talent for all uses, not merely scientists and engineers. This is vital because a major key to our Nation's future development and progress, whether in relation to peace or to defense, is our supply of welleducated manpower.

Die besondere Betonung von Exzellenz sowie Qualität sollte den Vereinigten Staaten im Wettbewerb der Systeme zum Erfolg verhelfen. Auf die Frage, wie diese Art von Qualität zu messen und in der Folge zu erreichen sei, entwickelten die USamerikanischen Bildungsplaner - hier in Form von Admiral Rickover - Vorschläge zur Umstrukturierung des Bildungssektors. Rickover und seine ideologischen Mitstreiter setzten dabei massiv auf Standardisierung und externe Kontrolle von Bildungsinstitutionen, wie der nachstehende Auszug zeigt:

It would be best to set up a private agency, a Council of Scholars, financed by our colleges and universities [...]. This council would set a national standard for the high school diploma, as well as for the scholastic competence of teachers. High schools accepting this standard would receive official accreditation, somewhat in the order of the accreditation given medical schools and hospitals (Rickover 1959b, S. 248).

In den Folgejahren versuchten die Bildungsplaner der Vereinigten Staaten Standards durch monetäre Anreize zu setzen. Da der Bildungssektor jedoch - ähnlich wie in Deutschland - Sache des Bundeslandes bzw. Bundesstaates in den USA war (sowie immer noch ist), nutzten die Schulen das Geld weitgehend ungeachtet der bildungspolitischen Zielvorgaben nach ihren eigenen Vorstellungen (vgl. Tröhler 2016). Als Reaktion auf diesen (Fehl-)Anreiz etablierten die Bildungsplaner Insti- 
tutionen, die Leistungsmessung durchführen und damit die erfolgreiche Umsetzung der Vorgaben überprüfen sollten. Monetäre Unterstützung für die jeweilige Einzelschule koppelten die US-amerikanischen Bildungsplaner an die Schülerergebnisse, die im Rahmen von Vergleichstests erhoben wurden. Hierdurch wandelte sich der Bildungssektor in den USA von einem input- zu einem outputgesteuerten System. Eine der zentralen Veränderungen, die dieser Wandel mit sich brachte: Fortan müssen Investitionen in die verschiedenen Bereiche des Systems geplant, verwaltet und evaluiert werden (vgl. King 2006, S. 175). Die ersten standardisierten und vergleichenden Tests - als Manifestation des Wandels von Input- zu Outputsteuerung wurden erstmals 1969 durchgeführt, ,allerdings noch auf freiwilliger Basis; die in diesem Zusammenhang entwickelten Testinstrumente wurden später zur Grundlage von PISA“ (Tröhler 2016, S. 102/103).

Im Jahr 1983 wurden die im Rahmen der standardisierten Vergleichstests entwickelten Testinstrumente und Verfahrensweisen zunehmend zum internationalen Standard ausgeweitet. Dies geschah indem die USA die OECD zunehmend dazu anhielt, die Outputorientierung von Bildungsprozessen zum neuen, globalen Standard zu erheben. Zentrales Organ für die globale Umgestaltung des Bildungssektors war das Center for Educational Research Innovation (CERI), denn ,,[ü]ber das CERI sollte also die globale Reformation der nationalen Bildungswesen nach dem Vorbild der US-amerikanischen Bildungspolitik erfolgen [...]“, und ,kurze Zeit später folgte dann, der selben Logik verpflichtet, PISA“ (beide Tröhler 2016, S. 103). Dieser Logik folgend konnten nun über international etablierte Standards die Outputs des Bildungssystems gemessen, überwacht und ggf. beeinflusst werden. Bildung und deren Outputs sollten vorhersagbar, rationalisierbar und „,result-oriented“ (Eide 1990, S. 34) werden oder wie Bürgi und Tröhler (2018, S. 87) zusammenfassend attestieren: „Ends and performance (outputs) and their actual relation can be monitored and, with this, the plans (or models) permanently controlled".

Als Konsequenz dieser Entwicklungen, die auch in Deutschland ihren Niederschlag fand und 2000 zum PISA-Schock führten, spielen gegenwärtig (Bildungs-)Standards im (Fach-)Unterricht eine tragende Rolle. Sie geben die Ziele von Schule und Unterricht vor und gelten gleichzeitig als Gradmesser für gelungenen oder verfehlten Unterricht. Bildungsstandards sollen dabei ,auf Schulfächer bezogen [...] den wesentlichen Bildungsgehalt eines Faches widerspiegeln“ (Reiss 2004, S. 635). Folgt man diesem Ansatz, so gelangt man zu der Annahme, dass „,[d]ie gesellschaftliche Bedeutung und die innewohnende ,Logik' der ,Sache ' [...] folglich wesentliche Bezugspunkte für das didaktische Handeln" darstellen (Niethammer und Langner 2017, S. 67). Diese tendenzielle Sachorientierung der Fachdidaktiken lässt sich auch an der Verortung der Fachdidaktiken innerhalb der Universitäten ablesen. Zwar existieren - teils aufgrund historisch gewachsener Strukturen, teils aufgrund universitärer Sonderwege - Ausnahmen und Abweichungen, allerdings beschreibt Vollmer (2007, S. 85) hier einen Mehrheitstrend, wenn er folgendes feststellt:

In der Mehrzahl der Fälle definieren sich die Fachdidaktiken relativ fachnah bzw. als Teil des Faches selbst, d.h. sie tragen ihrerseits zur Fachausbildung bei und setzen sich in unmittelbarer Nähe zu den fachwissenschaftlichen Kolle- 
ginnen und Kollegen mit den Zielsetzungen, den Schwerpunkten, der Relevanz und der Dynamik des Fachgebietes und der wissenschaftlichen Disziplin direkt auseinander.

Es kann argumentiert werden, dass die starke fachliche (Selbst-)Verortung, gepaart mit einer ebenso starken fachlichen Outputorientierung im Sinne eines nachweisbaren Lernzuwachses, in Einklang mit dem US-amerikanischen Standardisierungsmodells stehen ${ }^{2}$. Als Folge werden die über PISA perpetuierten Standards gegenwärtig auch als Gradmesser im öffentlichen Diskurs über Bildung herangezogen, wie Ritter (2021) argumentiert:

Auch von der gesellschaftlichen Laien-Öffentlichkeit und von der Bildungspolitik werden Vermittlungsfragen bezogen auf die Fachinhalte, also die Gegenstandsfelder der Didaktik, als Gradmesser für den Erfolg der Institution Schule herangezogen, denen auch eine Bedeutung für die zukünftige Entwicklung der Gesellschaft, besonders mit Blick auf sozio-ökonomische Fragen, zugebilligt wird.

Die durch die PISA-Reformen seitens der Politik artikulierten Ansprüche an die Institution Schule und damit ebenso an die Fachdidaktiken, reduziert die Lehrperson und ihr Handeln - provokativ gesprochen - rein auf die Beeinflussung von LehrLern-Prozessen. Ziel ist es dabei herauszufinden, ,aus welchen Ausgangsbedingungen damit welche Produkte entstehen können“ (Parchmann 2013, S. 32). Noch näher am Rickover'schen Schema operieren Klieme und Rakoczy (vgl. 2008, S. 225), die (fach-)didaktisches Handeln in einem Input-Prozess-Output Schema darstellen und damit das Handeln von Lehrern - wie auch die Absichten und Ziele von Fachdidaktiken - darauf reduzierten, die Effizienz innerhalb ebendieser Phasen zu erhöhen.

Es bleibt also festzuhalten, dass sich die Fachdidaktiken - zumindest nach dem Jahr 2000 - zunehmend nach außen verantworten und legitimieren mussten; dies geschah und geschieht im Hinblick auf festgeschriebene Inhalte der Kernlernpläne, die wiederum über operationalisierbare Kompetenzen ${ }^{3}$ prüfbar und damit sichtbar und vergleichbar gemacht werden konnten. Die Ergebnisse solcher Überprüfungen können national wie auch international verglichen werden mit dem Ziel Benchmarks bzw. best practices zu isolieren und anschließend flächendeckend anzuwenden. Basierend auf den Erkenntnissen dieser Vergleiche und Analysen können einzelne Aspekte des Bildungssystems modifiziert werden, um die von außen festgeschrie-

\footnotetext{
${ }^{2}$ Dies soll nicht proklamieren, dass universitäre Fachdidaktiken sich als Reaktion auf US-amerikanische Bemühungen fachnah positionierten, sondern, dass die bereits bestehende - und historisch gewachsene fachliche Nähe gepaart mit den neu einsetzenden Standardisierungsbemühungen darin resultierte, dass die entsprechenden Fachdidaktiken sich, bewusst oder unbewusst, zunehmend in die von der OECD gewünschte Richtung entwickelten.

${ }^{3}$ Häufig wird mit Blick auf den Kompetenzbegriff von einer Ökonomisierung von Bildung gesprochen (vgl. Grigat 2012, S. 76). Der Kompetenzbegriff wurde allerdings vom Militär in die Bildungslandschaft eingeführt. Das Militär wiederum orientierte sich dabei stark an der Medizin bzw. der medizinischen Ausbildung. Alle drei Disziplinen - Medizin, Militär, Wirtschaft - teilen eine Effizienz- bzw. Rationalisierungslogik. Diese mag zwar im 20. und 21. Jahrhundert primär mit dem Gegenstand der Wirtschaft verzahnt sein bzw. wurde seitens der OECD später hinsichtlich wirtschaftlichen Fortschrittes instrumentalisiert (vgl. Bürgi 2017, S. 292), hat ihre Ursprünge allerdings nicht dort.
} 
benen Outputs zu generieren. Der Ursprung und die Intentionen bei der Etablierung dieses Systems erklären auch den Umstand, dass einige Schulfächer (bspw. Mathematik oder Naturwissenschaften) weniger Probleme mit dem von PISA induzierten Wandel hatten. Schließlich passten die US-amerikanischen Bildungsplaner - im Kontext des Kalten Krieges - die neu geschaffene Bezugsgröße den Bedürfnissen und Logiken dieser Fächer an, mit dem Ziel die Outputs in diesen Fachbereichen zu erhöhen.

\subsection{Inklusive Pädagogik}

Zunehmend gerät die Auseinandersetzung mit den Ungleichheiten produzierenden Paradoxien gesellschaftlicher Systeme kombiniert mit solchen Prozessen in den Blick, über die sie „Gewinner und Verlierer“ (Prengel 2018, S. 42) produzieren. Ungleichheiten verschiedenster Dimensionen werden dabei als Ausgangspunkt sowie Resultat solcher Prozesse gesehen. Zudem unterminieren sie - sofern ein zu großer Anteil der Gesellschaft davon betroffen sein sollte - die Legitimation des gesamten Gesellschaftssystems. Referenz solcher Ausführungen ist, bildungshistorisch betrachtet, die Öffnung des Bildungssystems für breitere Schichten der Gesellschaft ${ }^{4}$.

Die auch auf wissenschaftlicher Ebene vielseitig behandelte Verzahnung von Bildung, gesellschaftlicher Stellung und Ungleichheit ist auch ein Grund, weshalb der Begriff der Inklusion in den sozialwissenschaftlichen Disziplinen schon seit den 1970er-Jahren zur etablierten Größe avancierte (vgl. Stichweh 1997) und bereits damals theoretisch hochgradig anschlussfähig an die zeitgleich laufenden erziehungswissenschaftlichen Diskurse war. Ausgehend von der Idee einer meritokratischen Gesellschaft, die illegitime Hürden wie Standesvorteile und Adelsprivilegien abschaffen möchte, führen Reflexionen über Inklusion diese Sicht auf Gesellschaft konsequent fort und hinterfragen, welche weiterhin bestehenden Hürden als legitim angesehen werden können. Inklusion bezeichnet in diesem Kontext dann im Groben gesprochen die „Markierung von „Menschen“ als relevant für soziale Systeme“ (Fuchs 2016, S. 398). Zeitgleich muss sich diese Relevanz ebenso in den Verfahrensweisen eines Systems widerspiegeln. Dabei werden Defizite bzw. defizitäre Ergebnisse seltener ontologisch erklärt, ,sondern als ausgrenzender und diskriminierender Umgang mit Differenzen“ gedacht (Sturm 2016, S. 181) - das betreffende System war/ist nicht auf die exkludierte Gruppe eingestellt bzw. berücksichtigt deren Idiosynkrasien nicht.

Insbesondere im Verlauf der zweiten Hälfte des 20. Jahrhunderts gerieten mit Blick auf derartige inklusive Grundannahmen verschiedene exkludierte Gruppen in den Fokus der öffentlichen Wahrnehmung sowie der deutschsprachigen erziehungswissenschaftlichen Forschung, ohne dass diese Reflexionen auf der Ebene des Diskurses mit dem Terminus der Inklusion in Verbindung gebracht wurden. Diese Gruppen zeichneten sich dadurch aus, dass sie von der (angenommenen bzw. konstruierten) Normgesellschaft im defizitären Sinne abwichen. Auf der Suche nach

\footnotetext{
4 Spätestens seit dem 19. Jahrhundert nimmt die Relevanz von Bildung und die Ausgestaltung der assoziierten Institutionen für die soziale und ökonomische Position eines jeden Individuums bedeutend zu (vgl. Horlacher 2011, S. 40/41). Dieser Anspruch öffnete das Bildungssystem graduell für verschiedene Gruppen - ein Prozess, der bis heute andauert.
} 
den systemischen Hindernissen, die die Gruppen an erfolgreicher gesellschaftlicher Partizipation hinderten, entwickelten sich verschiedene Strömungen und (Sub-)Disziplinen, die sich entweder über die Zeit etablieren konnten, sich gewandelt bzw. (weiter-)entwickelt haben oder von einem größeren Diskurs subsumiert wurden; hier seien bspw. die (Sub-)Disziplinen der Heilpädagogik (vgl. Biewer 2017), Ausländerpädagogik, Migrationspädagogik oder interkulturelle Pädagogik ${ }^{5}$ genannt. Die Fülle an aus dieser Perspektive betrachteten Gruppen und disziplinären Ausformungen als Antwort auf den Umgang mit Vielfalt, aber auch auf (Ergebnis-)Ungleichheit, erklärt sowohl die verschiedensten disziplinären Bezeichnungen wie auch gleichzeitig den Umstand, dass ,nicht alle, die vom Umgang mit Heterogenität reden, dasselbe [meinen]“" (Schildmann 2017, S. 83/84).

Diese historisch nachzeichenbare Mannigfaltigkeit im Umgang mit Differenz spiegelt sich auf die Gegenwart bezogen auch in der Uneinheitlichkeit des Inklusionsbegriffes wider (vgl. Löser und Werning 2013). Während Inklusion in den 1990er-Jahren - hier noch jenseits breit angelegter Reflexionen - primär auf körper-

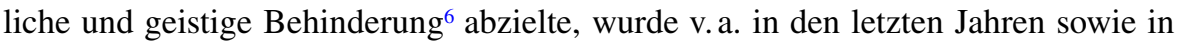
Kombination mit der vielseitig genährten Erweiterung der Inklusionsdebatte ,nicht [mehr, Anm. d. Verf.] ausschließlich auf die Differenzlinie der Behinderung“ fokussiert (Amrhein 2016, S. 19). Abhängig von der ausgewählten Position betrachtete Inklusion ,neben behinderten Kindern und Jugendlichen auch solche mit anderen Benachteiligungen [...]“" (Werning 2010, S. 284). Der rein auf körperlich bzw. geistige Behinderung abzielende Ansatz wird dabei häufig als eine Variante eines ,engen“ Inklusionsverständnisses gefasst und der multiperspektivische Zugang in der Konsequenz als ,weite' Variante bezeichnet (Löser und Werning 2015, S. 17) - ohne, dass hierüber im Diskurs Einheitlichkeit besteht.

Die mit der Inklusion verbundenen Werte wie die Fokussierung auf das Individuum, Abbau von illegitimen Hindernissen und Barrieren, Partizipation an gesellschaftlichen Prozessen manifestieren sich in inklusiver Pädagogik insoweit, als „dass (inklusive) Erziehung und Bildung nicht auf vorab festgestellten Unterschieden zwischen Individuen bzw. Gruppen basiert, sondern in einem ,non-kategorialen “ Sinne als Herausforderung für Diversität bzw. Heterogenität und als ,Miteinander der Verschiedenen" verstanden werden muss“ (Lindmeier und Lütje-Klose 2015, S. 8). Als zentrale Spezifika inklusiver Pädagogik werden von Textor (vgl. 2015, S. 27) die Einbeziehung aller Heterogenitätsdimensionen, eine systembezogene Sichtweise, rechtlicher Anspruch auf Inklusion sowie Dekategorisierung angeführt. Mit dem Verzicht auf Kategorisierung sollen ebenso die gruppenbezogene Ressourcenzuweisung verhindert und die individuell verschiedenen Bedarfslagen umfänglich berücksichtigt werden (vgl. ibid.). Eine derartige Ausrichtung des Inklusionsbegriffes und damit einhergehend ein entsprechendes Verständnis von inklusiver Pädagogik etabliert sich gegenwärtig auch sukzessive in Curricula, Lehrerbildungsverordnungen

\footnotetext{
5 Für einen exemplarischen Überblick über die Diskurslinien, die inhärenten Kritiken an ebendiesen und die Entwicklung im Bereich Migration und Pädagogik siehe auch Roth und Wolfgarten (2017).

6 Explizit sind hier die 1993 in Kraft getretene Änderung des deutschen Grundgesetzes sowie die 1994 verabschiedete Salamanca-Erklärung zu erwähnen. Beide nehmen im Umgang mit Vielfalt und Differenz explizit körperliche bzw. geistige Behinderung in den Fokus (vgl. Textor 2015).
} 
sowie nationalen wie internationalen Richtlinienpapieren und beschäftigt seitdem Fachwissenschaftler, Schulen, Praktiker und damit auch die Fachdidaktiken.

\section{Verdeckung als möglicher Umgang mit dem antinomischen Spannungsfeld}

Wie ausgeführt, orientierten sich - vor allem die in den internationalen Vergleichstests abgeprüften - Fachdidaktiken als Resultat bildungshistorisch nachzeichenbarer Prozesse im Rahmen der PISA-Reformen stark an dem Modell der Kompetenzorientierung, das wiederum primär fachliche Zugänge wählt. Die inklusive Pädagogik als zeitversetzt hinzugekommener Anspruch an die Fachdidaktiken sucht ihre Bezugsgröße primär im Individuum und betrachtet Strukturen hinsichtlich ihres Potenzials, individuelle Differenzen zu akkommodieren. Um die Möglichkeit der Verdeckung dieses entstehenden Spannungsfeldes in Betracht zu ziehen, wird in der Folge auf bereits erforschte Verdeckungskonzeptionen referiert, um deren generellen Mechanismus partiell zu skizzieren sowie ihre erziehungswissenschaftliche Relevanz und Anschlussfähigkeit zu umreißen.

Verdeckungsgeschehen sind bisher - unter verschiedenen terminologischen Bezeichnungen - in die unterschiedlichen, metatheoretischen Fachliteraturen eingegangen. Grundsätzlich kann festgehalten werden, dass Verdeckungsgeschehen meist dann zu beobachten sind, wenn artikulierte Werte mit realweltlichem Handeln kollidieren. Wocken (2010, S. 1) personifiziert diese Kollision im Bereich der Inklusion wie folgt: Es gibt Personen, die ,für Inklusion ein höfliches Lippenbekenntnis erübrigen können, aber an der weiteren Existenz von Sonderschulen unverbrüchlich festhalten“. Verdeckungsgeschehen als möglicher Umgang mit Dissonanzen sind interdisziplinär anschlussfähig und wurden in ihren Teilaufkommen bereits von verschiedenen Wissenschaftlern und Philosophen beobachtet und beschrieben, wobei eine Metatheoretisierung bislang ausgeblieben ist. Auf der Individualebene sind hier besonders Arbeiten der Psychoanalytik bzw. der Psychologie zu nennen, die im Latenten bzw. Verdrängten (vgl. Freud 2015; vgl. Adler 1930) oder in Inkongruenz (vgl. Rogers 1957) Gründe für Unwohlsein und/oder psychische Erkrankung ausmachen. Darüber hinaus konzeptualisiert Festinger (vgl. 1957) mit dem Phänomen der „kognitiven Dissonanz“ bereits eine, auf der Individualebene stattfindende, Version des Verdeckungsgeschehens.

Auf der Institutionenebene kann bspw. das von Foucault (2015, S. 8) - mit Blick auf Psychiatrien als Orte der Verdeckung - attestierte „Schweigen“ als eine Manifestation von Verdeckung gelesen werden. Für den Spezialfall, dass eine herrschende Ordnung von Neuankömmlingen hinterfragt bzw. irritiert wird, argumentiert Rancière (2018, S. 10), dass es zu einem „Unvernehmen“ kommen kann, wobei aufgrund der unausgewogenen Machtpositionen bzw. der im System inkludierten oder exkludierten Stellung - letztere nennt Rancière die „Anteillosen“ - mindestens eine Perspektive verdeckt bzw. nicht wahrgenommen wird ${ }^{7}$. Die Beobachtungen auf der

\footnotetext{
7 Konzeptionen dieser Art können - in ihrer extremsten Ausformung - in verdeckenden Systemen der „Archi-Politik“ (vgl. Rancière 2018) oder „Post-Politik“ (vgl. Heil 2019, S. 450) resultieren.
} 
Individual-, Institutionen- und Systemebene legen nahe, dass Verdeckung einen gängigen und häufig gebrauchten Mechanismus darstellt, der - aufgrund seiner Tendenz sich unsichtbar zu machen - selten konzeptualisiert bzw. in Erwägung gezogen wird.

Verdeckungsgeschehen sind daher als Prozesse, Mechanismen und Vorgehen zu rahmen, die von verschiedenen Akteuren und Institutionen initiiert werden können, um - unter Berücksichtigung vielfältiger Motivationen - Differenzen, bspw. von artikulierten und realisierten Werten, unsichtbar zu machen. Die Konzeptualisierung des Aufeinandertreffens von inklusiven Ansprüchen und kompetenzorientierter Didaktik als Verdeckungsgeschehen wäre insofern anschlussfähig, als dass bereits Fuchs (2016, S. 399) feststellte, dass ,Inklusionsbemühungen wie Substitute ,eigentlicher' Relevanzmarkierungen wirken, dass also immer ein Verdacht des ALS-OB mitläuft". So kann argumentiert werden, dass Inklusion von, in den Fachdidaktiken etablierten, intellektuellen Strömungen wie der Kompetenzorientierung möglicherweise umgedeutet und damit in ihrer ursprünglichen Form verdeckt worden sein könnte. Inklusion wird damit, Laclau (1996, S. 36ff.) folgend, zu einer ,leeren Signifikanten ${ }^{8 *}$.

Beide Ansprüche - Inklusion und kompetenzorientiertes Lernen - an den Schulsektor und damit auch an die Fachdidaktiken sind gleichermaßen relevant und rechtlich ähnlich verpflichtend. In der Folge werden mit der Betrachtung der Kategorien Teilhabe, Leistung und Perspektive exemplarisch drei konkrete Kristallisationspunkte dieses Spannungsfeldes charakterisiert und hinsichtlich ihrer antinomischen Ansprüche hin untersucht. Während Ritter (2021) die Annäherung der beiden Diskurse aneinander als Lösung des Problems versteht, stellt dieser Beitrag mit der Einführung des Verdeckungsgeschehens eine alternative Lesart der Geschehnisse zur Diskussion und führt diese - angewendet auf die drei o.g. Bereiche - aus.

\subsection{Teilhabe}

Bei der grundlegenden Frage der Teilhabe prallen die beiden umrissenen Argumentationsfiguren insofern aufeinander, als dass sie sich auf unterschiedliche Bezugsgrößen beziehen und somit zu unterschiedlichen Einschätzungen bzgl. Teilhabe bzw. der Negation ebendieser kommen. Als zentrale Bezugsgröße pädagogischer Überlegungen kann die Relation von Akteuren sowie deren Beziehung zueinander genannt werden, während didaktische Zugänge primär fachliche Interessen betonen (vgl. Ritter 2021). Diese divergierenden Bezugsgrößen manifestieren sich anteilig in andersgearteten Vorstellungen von Teilhabe, wie Ritter (2021) folgend ausführt: „Teilhabe im pädagogischen Sinne bezieht sich also auf soziale und institutionelle Aspekte, Teilhabe im didaktischen Sinne bezieht sich auf fachinhaltliche Normstrukturen und deren gesellschaftliche Relevanz". Die Ausgestaltung von Teilhabe, sodass sie sowohl fachliche wie auch pädagogische Ideale berücksichtigt und damit

\footnotetext{
${ }^{8}$ Laclau (vgl. 1996) argumentiert, dass leere Signifikanten auftreten, wenn im System etablierte Akteure Inhalte oder Probleme von marginalisierten Gruppen aufgreifen und diese versuchen im System umzusetzen, zu etablieren bzw. zu lösen. Die Unvereinbarkeit dieser beiden Stellungen (inkludiert/exkludiert) und der assoziierten Inhalte resultiert in leeren Signifikanten - Worten, die zwar oberflächlich das Thema oder Problem behandeln, bei genauerer Betrachtung lediglich geringe Schnittmengen mit dem Ausgangsgegenstand aufweisen.
} 
vereint, beurteilen einige Beobachter als ein unmögliches Unterfangen. So schreibt bspw. Feuser (2018, S. 117): „Es gibt keine inklusive Mathematik, keine inklusive Fremdsprache oder Geographie, keine inklusive politische Bildung oder Didaktik“ und referenziert in der Folge auf das in diesem Beitrag thematisierte Spannungsfeld, wenn er attestiert, dass ,immer noch Fächer, anstatt Menschen unterrichtet [werden]" (ibid.). Hinsichtlich der Frage, ob Fächer oder Individuen im Fokus stehen sollten, weist ebenso Schroeder (2017, S. 262) auf die Unvereinbarkeit der beiden Ansätze hin und betont die Möglichkeit der Auflösung fachbezogener Grenzen:

Ein transnormalistisches Bildungsverständnis als Ausgangspunkt einer inklusiven Didaktik würde hingegen auch die völlige Auflösung fachbezogener Grenzen im Sinne fachlicher Normalität implizieren. Inklusionsorientierte Ansätze aus fachdidaktischer Perspektive bleiben jedoch über ihren Gegenstand immer in eine flexibel-normalistische Strategie rückgebunden. So kann inklusive Didaktik in ihrer allgemeinen Formulierung zwar ein transnormalistisches Bildungsideal anstreben, sieht sich jedoch in der Einbindung fachdidaktischer Ansätze mit fachlichen Normalitätsgrenzen konfrontiert.

Auf dieses Spannungsfeld und auf die Gefahr der Auflösung fachbezogener Grenzen - damit einhergehenden dem möglichen Ende des Primats der fachlichen Kompetenzorientierung - antworteten die kompetenzorientierten Strömungen innerhalb der Fachdidaktiken hypothetisch mit dem oberflächlichen Adaptieren inklusiven Vokabulars und produzieren hiermit potenziell eine Variante eines Verdeckungsgeschehens. Ein mögliches Ziel dieses Vorgehens könnte es gewesen sein, grundsätzlichere Diskussionen, die durch die inklusive Diskurserweiterung hätten entstehen können - bspw. die Sinnhaftigkeit von Fächergrenzen, Rolle der Lehrperson, Schüler-Lehrer-Verhältnis im Raum Schule etc. - zu verdecken. In loser Anlehnung an Foucault (vgl. 2015, S. 8) kann dieses Vorgehen als der - in der Fachdidaktik geführte - Monolog der bereits etablierten Kompetenzorientierung über die inklusive Pädagogik gedeutet werden, errichtet auf dem (teilweisen) Schweigen der inklusiven Pädagogik.

\subsection{Leistung}

Neben der Frage nach Teilhabe liegt ebenso im divergierenden Bildungs- bzw. Leistungsbegriff enormes Irritationspotenzial für die rein kompetenzorientierten Fachdidaktiken. Durch die Kontextualisierung und ideengeschichtliche Einordnung der beiden Denkfiguren ermöglicht die bildungshistorische Perspektive eine Betrachtungsweise, die die Zentralität von Leistung(smessung) für den hier skizzierten Konflikt anteilig erklären kann. Auf die basalste Ebene heruntergebrochen, kann gesagt werden, dass sich ,[d]ie unterschiedlichen Logiken von Inklusion (als Teilhabe fördernd und damit differenzvermeidend oder -verringernd) und schulischer Leistung (als im meritokratischen Begründungssystem differenzschaffend)“ nicht auflösen lassen (Bräu 2018, S. 217) und sich daher ebenso in den divergierenden Verständnissen von Leistung und Bildung widerspiegeln.

Hinsichtlich dieser Verständnisse von Leistung bzw. den Zielen von Bildung hat das im Rahmen dieses Beitrages skizzierte Spannungsfeld das Potenzial, die kom- 
petenzorientierten Fachdidaktiken an eine Grenze stoßen zu lassen. Die Erweiterung des fachlichen, kompetenzorientierten Leistungsbegriffes durch einen mehrperspektivischen Leistungsbegriff könnte dazu führen, dass ,,[d]ieser [mehrperspektivische; Anm. d. A.] Leistungsbegriff die dominierende Orientierung an kognitiven Leistungen und fachspezifischen Wissenszuwachs in Frage" (Hackbarth und Martens 2018, S. 195/196) stellen würde. Die bedingungslose Umsetzung von inklusiver Pädagogik würde damit den - bis 2009 primär kompetenzorientierten - Fachdidaktiken ihren zentralen Legitimationsgegenstand rauben. Zwar könnten die Fachdidaktiken „neue Wege in der Bestimmung von Fachlichkeit gehen“ (Hackbarth und Martens 2018, S. 198), dies könnte allerdings in einer externen Legitimationsproblematik resultieren, die ebenso die raison d'être der Fachdidaktiken unterminieren würde, da nicht-outputorientierte Leistung, ,im paradigmatischen Widerspruch zu den ökonomischen Orientierungen in der Bevölkerung"stünde (Esslinger-Hinz 2014, S. 152). Wie bildungshistorisch dargestellt werden konnte, legitimierten sich die Fachdidaktiken gegenüber der Politik und Öffentlichkeit seit den PISA-Reformen über ihren Leistungs- und Kompetenzbegriff. Durch die Verknappung des Leistungsbegriffes auf outputorientierte Aspekte verdecken die kompetenzorientierten Strömungen innerhalb der Fachdidaktiken, dass Leistung bzw. genauer gesagt Bildung, historisch gesprochen primär das Individuum in den Fokus genommen hat bzw. nehmen sollte ${ }^{9}$ (vgl. Vogt und Neuhaus im Druck) - ein Umstand, den die inklusive Pädagogik erneut betonte. Durch die Verdeckung der Diskussion um die Ziele, Adressaten, Profiteure und Ideale von Bildung kann vermutet werden, dass die kompetenzorientierten Fachdidaktiken „Heucheleikosten ${ }^{10 “}$ (vgl. Greenhill 2016) von sich und dem Schulsystem fernzuhalten versucht haben. Darüber hinaus soll die grundsätzliche Unmöglichkeit der Vereinbarkeit dieser beiden Ansätze hinsichtlich Leistung verdeckt werden, ,[d]enn genau genommen geht es bei der Idee von Inklusion in der Schule (im meritokratischen System) um die Herstellung von Leistungsdifferenzen unter der Bedingung, dass Differenzen zwischen den Schüler*innen keine Rolle spielen sollen“ (Bräu 2018, S. 213).

\subsection{Perspektive}

Basierend auf den divergierenden Vorstellungen von Leistung, Teilhabe und Schule im Allgemeinen fokussieren die im Rahmen dieses Beitrages präsentierten Argumentationsfiguren auf unterschiedliche Aspekte von Schule. Dies steht auch in engem Zusammenhang mit den unterschiedlichen bildungshistorischen Kontexten, mit denen die beiden Figuren verbunden sind und führt zu differenten Annahmen hinsichtlich des zentralen Faktors, an dem Wandel bzw. Veränderung stattfinden kann bzw. sollte. Ritter (2021) führt dazu aus:

\footnotetext{
9 Kompetenzorientiertes Lernen fokussiert auf wirtschaftliche Leistungsfähigkeit bzw. Verwertbarkeit von Individuen und deren Fähigkeiten (vgl. Grigat 2012) - eine Perspektive die diametral dem individuumszentrierten Bildungsbegriff gegenübersteht.

10 Greenhill (2016) konzeptualisiert Heucheleikosten als jenen Reputationsverlust, der entsteht, wenn artikulierte Werte im Gegensatz zur real realisierten Handlung stehen und dies öffentlich gemacht wird sozusagen das Sichtbarmachen eines vorherigen Verdeckungsgeschehens.
} 
Während der Blick auf die Schüler*innen eher den Barriereabbau und die Möglichkeiten einer (kompensatorischen) individualisierten Förderung aller (und besonders von Schüler*innen mit besonderen Verhaltens-, Lern- und Leistungsmerkmalen) auslotet, richtet ein Blick auf die soziale Ordnung des Unterrichts die Aufmerksamkeit auf die heterogenitätssensible und prinzipiell wertschätzende Strukturierung von Bildungsräumen.

Stark verallgemeinert sowie zugespitzt kann gesagt werden, dass eine kompetenzorientierte Lesart von Fachdidaktik primär die Passung von Lern- bzw. Bildungsinput und Adressaten in den Blick nimmt, mit dem Ziel den gewünschten Output - die Erreichung eines vorgegebenen Lernzieles oder den teilweisen Erwerb einer Kompetenz - zu generieren. Daraus ergeben sich, im Falle des Nicht-Erreichens eines Zieles, die Möglichkeiten den Input zu verändern/erhöhen (bspw. unterstützende Angebot) oder die Passung näher an den Fähigkeiten des Lernenden zu orientieren. Inklusionspädagogische Ansätze suchen hingegen seltener den Fehler in einzelnen Prozessen, Akteuren oder Inhalten, als dass sie die schulischen Strukturen - von der Makroebene (bspw. Zielsetzungen des Schulsystems) bis hin zu Mikroprozessen im Unterricht - hinsichtlich illegitimer Hürden befragen ${ }^{11}$. Damit divergieren die von den beiden Denkrichtungen vorgetragenen Perspektiven und weisen unterschiedliche Konzepte von erfolgreichen Lehr-Lern-Prozessen auf - eine Differenz, die sich ebenso in den zwei vorangegangenen Kristallisationspunkten niederschlägt. Durch die Übernahme genuin inklusiver Lingua seitens der kompetenzorientierten Strömungen innerhalb des Mikrokosmos Fachdidaktik soll die potenzielle Unvereinbarkeit dieser beiden Strömungen verdeckt und so - durch das Ausbleiben größerer Verwerfungen - das Primat der Kompetenzorientierung innerhalb der Fachdidaktiken gewahrt bleiben.

\section{Ausblick}

Wie dieser Beitrag hoffentlich zeigen konnte, ist das Spannungsfeld, in dem sich die Fachdidaktiken gegenwärtig positionieren müssen bzw. in dem sie sich entwickeln können, antinomischer Natur und erzeugt damit nicht zu unterschätzenden Druck auf und Ansprüche an die Fachdidaktiken. Während die graduelle Annäherung dieser beiden Perspektiven auf Unterricht innerhalb der Fachdidaktiken durchaus einen

\footnotetext{
11 Die vorliegende Betrachtung und Konzeptualisierung von Fachdidaktiken ist begrenzt und verallgemeinert stark. Dabei soll nicht unterschlagen werden, dass kompetenzorientierte Fachdidaktiken und inklusive Pädagogik Schnittmengen aufweisen, bspw. die methodische Strukturierung von Lerngegenständen oder das Ziel, die Passung zwischen Inhalten und Adressaten zu erhöhen, da beide stark auf das Individuum fokussieren. Hier muss allerdings zwischen der fachdidaktischen Perspektive (die Modifikation von unterrichtlichen Prozessen) und der kompetenzorientierten fachdidaktischen Perspektive unterschieden werden. Letztere modifiziert unterrichtliche Prozesse hinsichtlich der in Abschn. 2.1 beschriebenen Ziele. Während Fachdidaktiken bzw. die fachdidaktische Perspektive per se kompatibel mit inklusiver Pädagogik sind, ist dies die kompetenzorientierte Fachdidaktik lediglich bedingt. Wie dargelegt, manifestiert sich in den Fachdidaktiken das Ringen um die Ausrichtung ebendieser, wobei Inklusion hier auf Kompetenzorientierung triff. Aufgrund der geteilten fachdidaktischen Perspektive sind diese Strömungen anteilig überlappend, differieren allerdings, wie in Abschn. 3 ausgeführt, in zentralen Verständnissen und Kategorien.
} 
denkbaren Verlauf der Geschehnisse darstellt, so muss allerdings auch die prinzipielle Unvereinbarkeit und, wie in diesem Beitrag argumentiert, die Möglichkeit der Verdeckung als Reaktion an diese Ansprüche ge- und bedacht sowie reflektiert werden. Lediglich durch eine offene Diskussion und das in Betracht ziehen vielfältiger Möglichkeiten kann dies zielführend erfolgen. In diesem Sinne möchte dieser Beitrag v.a. dazu animieren, neue Denkrichtungen zu eruieren und Möglichkeiten für fortführende Forschung zu öffnen.

Funding Open Access funding enabled and organized by Projekt DEAL.

Open Access Dieser Artikel wird unter der Creative Commons Namensnennung 4.0 International Lizenz veröffentlicht, welche die Nutzung, Vervielfältigung, Bearbeitung, Verbreitung und Wiedergabe in jeglichem Medium und Format erlaubt, sofern Sie den/die ursprünglichen Autor(en) und die Quelle ordnungsgemäß nennen, einen Link zur Creative Commons Lizenz beifügen und angeben, ob Änderungen vorgenommen wurden.

Die in diesem Artikel enthaltenen Bilder und sonstiges Drittmaterial unterliegen ebenfalls der genannten Creative Commons Lizenz, sofern sich aus der Abbildungslegende nichts anderes ergibt. Sofern das betreffende Material nicht unter der genannten Creative Commons Lizenz steht und die betreffende Handlung nicht nach gesetzlichen Vorschriften erlaubt ist, ist für die oben aufgeführten Weiterverwendungen des Materials die Einwilligung des jeweiligen Rechteinhabers einzuholen.

Weitere Details zur Lizenz entnehmen Sie bitte der Lizenzinformation auf http://creativecommons.org/ licenses/by/4.0/deed.de.

\section{Literatur}

Adler, A. (1930). Lebenslüge und Verantwortlichkeit in der Neurose und Psychose. In Praxis und Theorie der Individual-Psychologie (S. 170-177). Berlin, Heidelberg: Springer.

Amrhein, B. (2016). Inklusion als Mehrebenenkonstellation - Anmerkungen zu Rekontextualisierungstendenzen in inklusiven Bildungsreformen. In B. Amrhein (Hrsg.), Diagnostik im Kontext inklusiver Bildung (S. 17-39). Kempten: Klinkhardt.

Biewer, G. (2017). Grundlagen der Heilpädagogik und inklusiven Pädagogik. Bad Heilbrunn: UTB, Julius Klinkhardt.

Bräu, K. (2018). Inklusion und Leistung. In T. Sturm \& M. Wagner-Willi (Hrsg.), Handbuch schulische Inklusion (S. 207-222). Opladen, Toronto: UTB/Verlag Barbara Budrich.

Bürgi, R. (2017). Engineering the free world: the emergence of the OECD as an actor in education policy, 1957-1972. In M. Leimgruber \& M. Schmelzer (Hrsg.), The OECD and the international political economy since 1948 (S. 285-309). London/New York: Palgrave Macmillan.

Bürgi, R., \& Tröhler, D. (2018). Producing the 'right kind of people'-The OECD indicators in the 1960s. In Lindblad, Pettersson \& Popkewitz (Hrsg.), Education by the Numbers and the Making of Society (S. 75-91). London.: Routledge.

Buss, D. C. (1980). The Ford Foundation in public education: Emergent patterns. In R. F. Arnove (Hrsg.), Philanthropy and cultural imperialism: the foundations at home and abroad (S. 331-361). Boston: G. K. Hall.

Creagh, E. (1957). "Nixon seemed more concerned over sputnik than president," Associated Press. https:// surveillancevalley.com/content/citations/ed-creagh-nixon-seemed-more-concerned-over-sputnikthan-president-associated-press-17-october-1957.pdf (Erstellt: 10.1957). Zugegriffen: 18.11.2020.

Eide, K. (1990). 30 Years of educational collaboration in the OECD. Paris: UNESCO.

Esslinger-Hinz, I. (2014). Inklusion und Leistung - Paradigmata im Widerspruch? In E.-K. Franz, S. Trumpa \& I. Esslinger-Hinz (Hrsg.), Inklusion - Eine Herausforderung für die Grundschulpädagogik (S. 142-154). Hohengehren: Schneider Verlag.

Festinger, L. (1957). A theory of cognitive dissonance. Stanford: Stanford University Press. 
Feuser, G. (2018). Momente der Ideengeschichte der Integration bzw. Inklusion im Feld der (Schul-)Pädagogik. In T. Sturm \& M. Wagner-Willi (Hrsg.), Handbuch schulische Inklusion (S. 111-126). Opladen/Toronto: Barbara Budrich Verlag, UTB.

Foucault, M. (2015). Wahnsinn und Gesellschaft. Eine Geschichte des Wahns im Zeitalter der Vernunft. Frankfurt am Main: Suhrkamp.

Freud, S. (2015). Die Traumdeutung. Hamburg: Nikol Verlag.

Fuchs, P. (2016). Inklusion/Exklusion - theoretische Präzisierung. In I. Hedderich, G. Biewer, J. Hollenweger \& R. Markowetz (Hrsg.), Handbuch Inklusion und Sonderpädagogik (S. 397-401). Bad Heilbrunn: Julius Klinkhardt/UTB.

Götz, M., Einsiedler, W., Miller, S., \& Vogt, M. (2016). Diskussionspapier zum Selbstverständnis der Grundschulpädagogik als wissenschaftliche Disziplin. https://www.dgfe.de/fileadmin/Ordner Redakteure/Sektionen/Sek05_SchPaed/GFPP/2016_Diskussionspapier_Grundschulpaedagogik.pdf. Zugegriffen: 09.12.2019.

Greenhill, K. M. (2016). Weapons of mass migration: forced displacement, coercion, and foreign policy. Ithaca: Cornell University Press.

Grigat, F. (2012). Die Kompetenzkatastrophe - oder „Die Wiederkehr der Bildungsphilister durch die Hintertür“. Pädagogische Korrespondenz, (46), 74-81.

Hackbarth, A., \& Martens, M. (2018). Inklusiver (Fach-)Unterricht: Befunde - Konzeptionen - Herausforderungen. In T. Sturm \& M. Wagner-Willi (Hrsg.), Handbuch schulische Inklusion (S. 191-206). Opladen, Toronto: Barbara Budrich Verlag, UTB.

Heil, R. (2019). Slavoj Žižek. In D. Comtesse (Hrsg.), Radikale Demokratietheorie. Ein Handbuch (S. 444-452).

Horlacher, R. (2011). Bildung. Bern: UTB, Haupt.

Hummrich, M., \& Kramer, R.-T. (2017). Was ist schulische Sozialisation? In Schulische Sozialisation. Basiswissen Sozialisation (Bd. 5, S. 7-27). Wiesbaden: Springer VS.

Jackson, P. W. (1968). Life in the classroom. New York: Holt, Rinehart and Winston.

King, A. (2006). Let the cat turn round: one man's traverse of the twentieth century. London: CPTM.

Klieme, E., \& Rakoczy, K. (2008). Empirische Unterrichtsforschung und Fachdidaktik - Outcome-orientierte Messung und Prozessqualität Unterrichts. Zeitschrift Für Pädagogik, 54, 222-237.

Laclau, E. (1996). Emancipation(s). London: Verso.

Lindmeier, C., \& Lütje-Klose, B. (2015). Inklusion als Querschnittsaufgabe in der Erziehungswissenschaft. Erziehungswissenschaft, 51, 7-16.

Löser, J.M., \& Werning, R. (2013). Inklusion aus internationaler Perspektive - ein Forschungsüberblick. Zeitschrift Für Grundschulforschung, 6(1), 21-33.

Löser, J. M., \& Werning, R. (2015). Inklusion - allgegenwärtig, kontrovers, diffus. Erziehungswissenschaft, 26, 17-24.

Niethammer, M., \& Langner, A. (2017). Inklusion als fachdidaktischer Anspruch. In J. Seifried, S. Seeber \& B. Ziegler (Hrsg.), Jahrbuch der berufs- und wirtschaftspädagogischen Forschung (S. 63-78). Opladen: Budrich Verlag.

Parchmann, I. (2013). Wissenschaft Fachdidaktik - Eine besondere Herausforderung. Beiträge Zur Lehrerinnen Und Lehrerbildung, 31-41.

Prengel, A. (2018). Pädagogik der Vielfalt. Inklusive Strömungen in der Sphäre spätmoderner Bildung. In F. J. Müller (Hrsg.), Blick zurück nach vorn - WegbereiterInnen der Inklusion. Dialektik der BeHinderung, (Bd. 2, S. 33-56). Gießen: Psychosozial-Verlag.

Rancière, J. (2018). Das Unvernehmen. Politik und Philosophie. Frankfurt am Main: Suhrkamp.

Reiss, K. (2004). Bildungsstandards und die Rolle der Fachdidaktik am Beispiel der Mathematik. Zeitschrift Für Pädagogik, 50, 635-649.

Rickover, H. G. (1959a). Education is our first line of defense-make it strong. In H. G. Rickover (Hrsg.), Education and freedom (S. 15-38). New York: Dutton \& Co.

Rickover, H. G. (1959b). Education and freedom (5. Aufl.). New York: Dutton \& Co.

Ritter, M. (2021). Strukturelle Disparitäten. Eine vergleichende Diskussion zur Konzeptualisierung des Inklusionsbegriffs in Pädagogik und Fachdidaktik. Zeitschrift für Grundschulforschung, 14(1).

Rogers, C. R. (1957). The necessary and sufficient conditions of therapeutic personality change. Journal of Consulting Psychology. https://doi.org/10.1037/0033-3204.44.3.240

Roth, H.J., \& Wolfgarten, T. (2017). Eine strategische Wende und ihre Erzählungen. Von der Ausländerpädagogik zur Interkulturellen Pädagogik. In S. Aßmann, P. Moormann, K. Nimmerfall \& M. Thomann (Hrsg.), Wenden (S. 185-199). Wiesbaden: Springer VS. 
Schildmann, U. (2017). Leistung in der inklusiven Pädagogik - normalismustheoretisch reflektiert. In B. Lütje-Klose, M.-A. Boger, B. Hopmann \& P. Neumann (Hrsg.), Leistung inklusive? - Inklusion in der Leistungsgesellschaft (Bd. 1, S. 83-90). Bad Heilbrunn: Julius Klinkhardt.

Schroeder, R. (2017). Didaktische Normalität? - Normalismustheoretische Betrachtungen im Zusammenhang von (Fach-)Didaktik und Inklusion. In B. Lütje-Klose, M.-A. Boger, B. Hopmann \& P. Neumann (Hrsg.), Leistung inklusive? - Inklusion in der Leistungsgesellschaft (Bd. 1, S. 83-90). Bad Heilbrunn: Julius Klinkhardt.

Stichweh, R. (1997). Inklusion/Exklusion und die Theorie der Weltgesellschaft. In K. S. Rehberg (Hrsg.), Differenz und Integration: Die Zukunft moderner Gesellschaften. Wiesbaden: VS.

Sturm, T. (2016). Phasen der Entwicklung Inklusiver Bildung. In I. Hedderich, G. Biewer, J. Hollenweger \& R. Markowetz (Hrsg.), Handbuch Inklusion und Sonderpädagogik (S. 179-183). Bad Heilbrunn: Julius Klinkhardt, UTB.

Textor, A. (2015). Einführung in die Inklusionspädagogik. Bad Heilbrunn: Verlag Julius Klinkhardt, UTB. Tröhler, D. (2016). Sputnik, die Pädagogisierung des Kalten Krieges und PISA. In K. Zierer, J. Kahlert \& M. Burchardt (Hrsg.), Die pädagogische Mitte. Plädoyers für Vernunft und Augenmaß in der Bildung (S. 97-108). Bad Heilbrunn: Klinkhardt.

Tröhler, D. (2019). Bildungsstandards oder die Neudefinition von Schule: Entstehung, Verbreitung und Folgen einer globalen bildungspolitischen Sprache. In Z. Zuber, H. Altrichter \& M. Heinrich (Hrsg.), Bildungsstandards zwischen Politik und schulischem Alltag. Educational Governance, (Bd. 42, S. 3-24). In: Springer.

Vogt, M., \& Neuhaus, T. (im Druck/2021). Self-Cultivation and the Concept of German Bildung. In M. A. Peters (Hrsg.), Moral Education and the ethics of self-cultivation. New York: Springer.

Vogt, M., Boger, M.-A., \& Bühler, P. (Hrsg.) (im Druck/2021). Inklusion als Chiffre - Bildungshistorische Analysen und Reflexionen. Bad Heilbrunn: Julius Klinkhardt.

Vollmer, J.H. (2007). Zur Situation der Fachdidaktik an deutschen Hochschulen. Erziehungswissenschaft, $18,85-103$.

Werning, R. (2010). Inklusion zwischen Innovation und Überforderung. Zeitschrift Für Heilpädagogik, 8, 284-291.

Wocken, H. (2010). Über Widersacher der Inklusion und ihre Gegenrede. APuZ, 23, 1-8. 